\title{
La confiance en éducation entre l'autorité et le pouvoir
}

Quelques réflexions sur les réalités éducatives au Portugal

Trust in education between authority and power. Reflections on educational realities in Portugal

La confianza en educación entre la autoridad y el poder. Algunas reflexiones sobre las realidades educativas en Portugal

Adalberto Dias de Carvalho et Nuno Fadigas

\section{OpenEdition}

\section{Journals}

Édition électronique

URL : https://journals.openedition.org/ries/5505

DOI : 10.4000/ries.5505

ISSN : 2261-4265

\section{Éditeur}

France Education international

Édition imprimée

Date de publication : 1 septembre 2016

Pagination : 49-55

ISBN : 978-2-85420-611-1

ISSN : $1254-4590$

Référence électronique

Adalberto Dias de Carvalho et Nuno Fadigas, « La confiance en éducation entre l'autorité et le pouvoir », Revue internationale d'éducation de Sèvres [En ligne], 72 I septembre 2016, mis en ligne le 01 septembre 2018, consulté le 01 juillet 2021. URL : http://journals.openedition.org/ries/5505; DOI : https://doi.org/10.4000/ries.5505 


\title{
La confiance \\ en éducation entre \\ l'autorité et le pouvoir
}

\section{Quelques réflexions sur les réalités éducatives au Portugal}

\author{
Adalberto Dias de Carvalho \\ Institut de philosophie de l'Université de Porto \\ Nuno Fadigas \\ Institut de philosophie de l'Université de Porto
}

\section{Autorité et POUVOIR}

Chaque fois qu'on se propose de réfléchir à la problématique de la confiance à l'école en particulier et d'une manière générale en éducation, on pose d'emblée la question du rapport entre les notions et les pratiques qui y sont liées et les concepts et actions qui ont directement à voir avec l'exercice de l'autorité et du pouvoir.

Dans un article antérieur (Dias de Carvalho, 2010), en partant des considérations d'Alain Renaut développées dans son ouvrage La fin de l'autorité, nous avions justement posé comme centrale, lorsqu'il s'agit des relations éducatives ou, plus largement, sociales, la dialectique qui se joue entre une autorité traditionnelle en crise, en tant que dispositif régulateur de l'ordre appuyé sur une hiérarchie et sur un fondement a priori, et les exigences contemporaines inhérentes aux critères démocratiques. Dans le cas d'une autorité attachée à une pratique de pouvoir abstrait et inconditionnel, il y a, d'après Renaut, risque d'émergence d'un sur-pouvoir, ce qui réduit les possibles manifestations d'autonomie. Cependant, à l'inverse, toujours d'après cet auteur, si, dans la "décomposition des formes traditionnelles de l'autorité », l'époque moderne regrette l'émiettement de ces expressions de l'autorité, elle réclame de manière implicite leur retour, mais du fait des repères démocratiques dominants dans nos sociétés, elle ne peut plus les accepter sans un contrôle critique du côté éducatif, énoncé au nom de la dignité des enfants : au nom de leurs droits. Nous sommes alors au cœur du débat qui interroge les apparentes ou potentielles contradictions entre la proclamation des «droits-liberté » et celle de la défense des «droits-protection" des enfants, institués tous les deux par la Convention des droits de l'enfant. En fait, notre époque hésite entre, d'un côté, la reconnaissance première de la fragilité des enfants qui, en justifiant une attitude protectrice de la part des enseignants et des parents, pourrait risquer de donner lieu à des 
dérives autoritaires, et, de l'autre, l'affirmation prioritaire de l'autonomie des enfants, avec tous les dangers inhérents à une perte ou à un affaiblissement de l'autorité elle-même et une dérive spontanée du droit d'initiative des enfants. Avec une reconnaissance exigeante de toutes les dimensions des droits de l'enfant, il faudra finalement trouver les voies d'articulation cohérente entre ces deux formes de droits car elles sont - ou devront être - complémentaires.

Il dépend des éducateurs (au sens où cela relève du pouvoir qui est le leur) de savoir, situation par situation, comment faire en sorte que la prise en compte d'un des deux groupes de droits prémunisse contre les conséquences d'une reconnaissance exclusive de l'autre, et réciproquement. (Renaut, 2004).

La confiance pourrait jouer un rôle dans cette articulation. L'autorité éducative, il ne faut jamais l'oublier, doit être au service des enfants et s'il y a un pouvoir - des enseignants ou des parents -, ce pouvoir sera toujours, par son statut anthropologique, un pouvoir transitoire, dont la finalité, à la différence $\mathrm{du}$ pouvoir politique, se réalisera seulement dans la mesure où son exercice vise et réalise son dépassement : en faveur de la réalisation de l'enfant comme une personne de droits.

\section{LE CONCEPT DE CONFIANCE}

Le Larousse définit la confiance comme un «sentiment de sécurité d'une personne à l'égard de quelqu'un ou de quelque chose ", par opposition à la défiance et à la méfiance.

Nuno Ferreira (2014), à la suite d'autres travaux antérieurs, nous rappelle qu'il y a des rapports entre la relation pédagogique, notamment dans la classe, et d'autres types de sociabilité au niveau de la famille, des réseaux sociaux virtuels, des amis, etc., où des aspects comme l'autonomie et la confiance deviennent importants. À partir de cela, en détachant le rôle des technologies de la communication (TIC) en ce qui concerne les gains d'autonomie par les jeunes à travers le renforcement de l'accès à l'information et des possibilités d'initiatives personnelles, il présente la relation pédagogique comme le principal moteur du jeu interactif entre les règles et l'autorité d'un côté et les attitudes des élèves de l'autre, jeu où la confiance est fondamentale pour l'exercice de l'autorité elle-même. Par ailleurs, soit l'autonomie soit la confiance interviennent aussi dans d'autres logiques d'action extérieures à l'école et moins assujetties à l'imposition des normes du cadre institutionnel. Après avoir présenté les résultats d'une étude empirique menée auprès de différents groupes d'élèves distingués selon leurs degrés de confiance et d'autonomie, l'auteur conclut que l'autonomie est plus manifeste dans les groupes où la confiance et la proximité avec l'enseignant sont plus grandes. Dans le même temps, ces élèves acceptent plus facilement les règles telles que l'absence des portables, quoique en profitant de leur utilisation différemment d'autres groupes, où moins d'autonomie représente moins de confiance et vice-versa. 
Dans un autre article, Michela Marzano (2010) souligne que la confiance, lorsqu'elle existe, représente d'emblée, d'un côté, la capacité à créer des liens en rendant possible la socialité et le fonctionnement de la démocratie, et, de l'autre, la vulnérabilité qui nous caractérise, nous obligeant à accepter le risque de la dépendance, le risque d'être trahi par celui qui a été dépositaire de notre confiance :

La confiance est liée à la nature même de l'existence humaine, au fait que nous ne sommes jamais complètement indépendants des autres et autosuffisants, même lorsque nous avons la possibilité d'atteindre un certain degré d'autonomie morale.

À cause de cela, nous sommes souvent contraints d'accepter de dépendre de la bienveillance des autres, ce qui est plus évident pendant l'enfance, moment où justement l'autonomie se construit à travers l'acceptation de l'exposition de la fragilité devant les adultes auxquels l'enfant fait confiance. En effet, il semble que les êtres humains ont besoin de stabilité, en souhaitant pour cela " pouvoir compter sur les autres, prévoir leurs comportements et avoir des points de repère » (Marzano, 2010). La confiance devient alors condition de l'espérance...

Ces conclusions apparaissent comme des remarques fondamentales lorsqu'on veut bien mener les procédures et les processus éducatifs avec les élèves au cours du passage de l'enfance à la jeunesse, c'est-à-dire, pendant la transition de la dialectique liberté-protection, dominante dans la première phase, vers celle de l'autonomie-confiance qui, en étant toujours latente, au moins apparemment, tend à oublier la protection, que les jeunes refusent cependant en tant qu'elle peut signifier leur hétéronomie.

Pourtant, si ces questions se posent au plan de la formation de l'identité psychologique et personnelle des destinataires de l'action éducative dans des contextes scolaires et familiaux, voilà qu'elles sont également soulevées quand on essaie de comprendre ce qui se passe au niveau des préoccupations politiques des systèmes éducatifs et de la formation de la citoyenneté. Alors la participation comme concept-clé et comme pratique souhaitée tend à occuper la première place des propositions.

\section{LA PLACE INSTITUTION NELLE DE LA CONFIANCE DANS LE SYSTÈME ÉDUCATIF PORTUGAIS}

Dans le cas portugais, il est intéressant de mentionner, à titre d'illustration, la recommandation $\mathrm{n}^{\circ} 3 / 1998$ du Conseil national d'éducation,

La pleine réalisation de la mission de former pour une citoyenneté responsable n'existe pas si les institutions éducatives continuent à " enseigner » les règles de la vie démocratique et à organiser simultanément les écoles en contradiction avec les principes et les normes qu'elles enseignent. 
Cette affirmation est immédiatement suivie de l'observation suivante : Souvent les jeunes de ce niveau ne sont plus stimulés en vue de la participation et de l'exercice concret des responsabilités au sein de la vie scolaire elle-même.

Cette exigence de participation témoigne de la reconnaissance de la primauté de l'implication des élèves dans le quotidien de la vie scolaire comme sujets et non comme simples objets de l'action des enseignants, et d'autant plus dans ce cas, car il s'agit de l'apprentissage de comportements sociaux où l'assomption de la responsabilité est un présupposé inaliénable. Or la participation et la responsabilité présupposent, à leur tour, la confiance en soi et dans les autres. En fait, il y a "convivialité » ${ }^{1}$ démocratique seulement dans la mesure où les personnes se reconnaissent comme des êtres de relation et comme des citoyens à part entière, capables d'initiatives et capables d'accueillir celles des autres, ce qui implique de détenir les capacités créatrices et de jugement critique nécessaires pour gérer d'une manière harmonieuse cette ouverture à autrui, qui est le pivot de la confiance. La confiance passe ainsi par une intersubjectivité qui peut déboucher sur l'institutionnalisation de contrats de coopération.

Au Portugal, l'Assemblée de la République a approuvé, le 5 septembre 2012, une nouvelle version du statut de l'élève qui définit notamment les droits et les devoirs de celui-ci. L'idée de la promotion d'une culture de la citoyenneté, au nom des valeurs de la dignité humaine, de la démocratie, de l'exercice responsable de la liberté humaine et de l'identité nationale, acquiert dans ce document une prépondérance remarquable. De ces principes découlent, entre autres, le droit de chaque élève à bénéficier d'un projet éducatif qui lui offre les conditions nécessaires à son développement physique, intellectuel, moral, culturel et civique, ainsi que le droit d'intervenir dans l'administration de l'école, de se réunir et d'élire ses représentants, en respectant les normes et l'autorité des enseignants ainsi que des autres personnels de l'établissement.

On reconnaît, dans ce type de documents, tout le poids d'une rhétorique politique qui met en ouvre les conditions législatives pour la création d'un climat de confiance à l'école, comme l'exige d'ailleurs la participation à une culture démocratique. Cependant le mot "confiance " demeure absent... Il faut dire aussi que, fréquemment, l'on assiste à des reculs importants dans ce domaine, en raison de problèmes disciplinaires graves qui justifient qu'on réclame un renforcement de l'autorité des enseignants et un appel à la responsabilité des parents.

1. Convivência, en portugais. (ndlr) 


\section{LE CONCEPT DE CONFIANCE AU SEIN DES DÉBATS ET DES PRATIQUES PÉDAgOGIQUES}

La confiance implique la recherche d'une certaine sécurité dans le futur, et ainsi la construction de l'avenir comme un projet. Ce projet entraîne à son tour un engagement personnel et collectif, qui tend à faire des événements des avènements marqués par un certain degré de prévision, en accord avec ce que l'on souhaite. D'ailleurs, la capacité à idéaliser et à mettre en œuvre des projets s'avère comme une manifestation de l'élan de vivre, et d'envisager l'accomplissement de ces projets comme l'une des perspectives de réalisation d'un bonheur utopique ou qui manque, tout simplement, et que le présent n'arrive jamais à procurer. Chercher le bonheur témoigne du fait d'avoir confiance dans un futur qu'on n'attend pas passivement, mais dont on espère plutôt être, dans une certaine mesure, l'auteur ou le co-auteur, et même mieux, le protagoniste et pas simplement le figurant.

Le «travail par projet» a été inspiré par ces propos éducatifs en gagnant même le droit de faire partie des orientations des textes officiels.

En effet, un texte de 1989, le décret-loi $n^{\circ} 286 / 89$, institue ce qu'on a appelé l' "aire école », en vue de la concrétisation, dans le cadre de l'autonomie des écoles, de projets multidisciplinaires. Plus tard, un autre texte officiel datant de 1990, l'arrêté n 142ME/90, a précisé en détail les finalités de "l'aire école », en indiquant notamment ceci :

(...) la concrétisation d'activités susceptibles de promouvoir le développement de l'esprit d'initiative, d'organisation, d'autonomie et de solidarité, aspects fondamentaux pour la formation intégrale de l'élève.

L'initiative de ces projets appartient aux écoles ou tout simplement aux enseignants et aux élèves. Avec l'accompagnement des enseignants et en utilisant la méthode de travail par projet, c'est aux élèves et à leur initiative, selon ce texte légal, qu'est confiée la responsabilité de choisir, partager, exécuter et autoévaluer les tâches à réaliser.

Mais voilà que, après une période d'intenses débats où les adversaires de cette réforme se sont engagés dans une défense de l'enseignement des contenus scientifiques qu'ils estimaient méprisés, et aussi en raison d'un manque de ressources pour mettre en œuvre de façon cohérente l'aire école, un autre décretloi $\left(n^{\circ} 18,2011\right)$ a mis fin à cet espace non disciplinaire...

Il est évident que, de cette manière, prend fin également une période soutenue par l'éducation nationale, pendant laquelle un certain recul, au moins formel, de l'autorité traditionnelle des enseignants, quoique seulement dans une niche pédagogique restreinte, favorisait l'initiative des élèves fondée sur un contrat de confiance entre eux, les enseignants et l'école d'une manière générale. 
Cependant, depuis 1987 et à la suite d'un projet de la Commission de réforme du système éducatif, se développe en parallèle dans un certain nombre d'écoles officielles et privées, en tant qu'expérience pédagogique reconnue et acceptée par l'État, le mouvement de l' "Escola cultural». Ce mouvement a tiré ses racines philosophiques du concept d'anthropagogie défini par Manuel Patrício (1990) comme une "science pratique et normative en vue de la formation de l'homme dans la pureté et la plénitude de son humanité », ce qui implique l'acceptation de la "polydimensionnalité axiologique de l'homme » et de l'activité humaine, dans son essence, comme une activité culturelle créatrice et multiple. À la différence du rationalisme dogmatique de l'école traditionnelle, qui impose, comme fondement de l'autorité, la prévalence pédagogique et hétéronome du patrimoine culturel, à la différence également de l'individualisme de l'École nouvelle, qui essaie d'écraser le binôme autorité-pouvoir, le mouvement de l'École culturelle défend l'idée d'une école conçue comme une organisation pédagogique centrée sur la personne de l'élève en tant que personne autonome qui, avec l'engagement des enseignants, bénéficie, au sein d'une communauté éducative non strictement curriculaire, de la réalisation toujours participative d'un tissu riche et complexe d'activités culturelles et sportives, dans lequel la confiance est la clé de toutes les initiatives et relations.

Ce mouvement s'est relativement fortement développé sur le terrain pendant une dizaine d'années, et il continue à s'affirmer comme un repère pédagogique, en ce qui concerne l'histoire récente des idées pédagogiques au Portugal.

Aujourd'hui, l'Escola da Ponte, près de Porto, présente des caractéristiques très particulières. Elle est fondée d'une manière très évidente sur le principe du contrat pédagogique qui reconnaît aux enfants et aux jeunes le droit à une organisation éducative fortement communautaire, dans laquelle les élèves ont un grand champ d'initiatives et d'auto et hétéro-évaluation de tout ce qui se passe en matière d'espaces, de temps, de contenus et de stratégies scolaires. La solidarité, l'autonomie et la responsabilité constituent ses repères fondamentaux, en se réclamant, dans ce cadre, du mouvement de l'École moderne inspiré par les propos pédagogiques de Célestin Freinet.

De toute façon, en ce qui concerne les écoles du système éducatif en général, par exemple, la discipline appelée "aire d'intégration », transversale dans l'enseignement professionnel, et la discipline de philosophie privilégient, au Portugal, des thèmes ou problèmes qui, à la suite de négociations en classe, sont choisis en fonction des intérêts des élèves. Par ailleurs, les délégués de classe élus sont membres effectifs des conseils de classe, pouvant ainsi intervenir dans les débats qui se posent dans cette structure intermédiaire de gestion scolaire. L'exercice de l'autorité, démocratisé, est de plus en plus partagé. D’ailleurs, comme le dit Onora O’Neill, le bon gouvernement des institutions est possible simplement si l'on permet des formes d'auto-gouvernement adaptées à des tâches plus restreintes. Cela, signifierait, dans ce cas, l'absence d'évaluations externes 
permanentes qui, à cause de la préoccupation liée au contrôle constant des activités, détournent finalement les professionnels d'un exercice responsable de leurs fonctions et d'une attention aux besoins et intérêts de leurs élèves.

Il est aussi intéressant de constater que, au niveau des manuels scolaires, les activités proposées, qui réclament l'initiative des élèves, se sont remarquablement développées : alors qu'elles étaient, dans certains cas, inexistantes en 1975, on en dénombre 1000 environ en 2014.

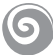

Il est évident que la problématique de la confiance soulève la question éducative de la promotion de l'autonomie par rapport à celle de l'autorité et du pouvoir. Souvent, l'autorité traditionnelle des enseignants se sent menacée devant les contrats scolaires formels ou tacites qui valorisent la participation active des élèves. Pourtant, il n'y a pas de démocratie en l'absence de projets éducatifs de construction d'une citoyenneté d'implication et d'engagement. Contre les risques de la dichotomie autorité/pouvoir, la confiance, valorisée comme intermédiaire opérationnel entre ces deux catégories, trouve, par la promotion de contrats de formation, son sens anthropologique, son statut épistémologique et son rôle pédagogique.

\section{BIBLIOGRAPHIE}

DIAS DE CARVALHO A. (2010) : "Autorité et pouvoir dans l'éducation contemporaine : un pouvoir sans autorité est-il possible? », Penser l'éducation, $\mathrm{n}^{\circ} 28$, décembre, p. $42-50$.

FERREIRA N. (2014) : «Autonomia, autoridade e confiança em tempo de novas TIC : atitudes e práticas diferenciadas entre os alunos do secundário ", Sociologia, vol. 27, Porto, janvier, p. 111-141.

MARZANO M. (2010) : "Qu'est-ce que la confiance?», in Études I, tome 412, p. 53-63.

O’NEILL O. (2002) : «A Question of Trust: The BBC Reith Lectures 2002 », Cambridge University Press, Cambridge [en ligne] http://goo.gl/fwTxt2, consulté le 9 juin 2016.

PATRÍCIO FERREIRA M. (1990) : Escola Cultural, Lisboa, Texto Editora.

RENAUT A. (2004) : La fin de l'autorité, Paris, Flammarion. 
